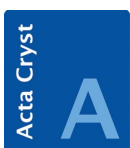

FOUNDATIONS ADVANCES
Received 9 June 2015

Accepted 5 August 2015

Edited by J.-G. Eon, Universidade Federal do Rio de Janeiro, Brazil

Keywords: periodic entanglement; networks; hyperbolic reticulations; tilings; crystal nets; frameworks; Borromean nets; surfaces; graphs; catenation; enumeration

Supporting information: this article has supporting information at journals.iucr.org/a

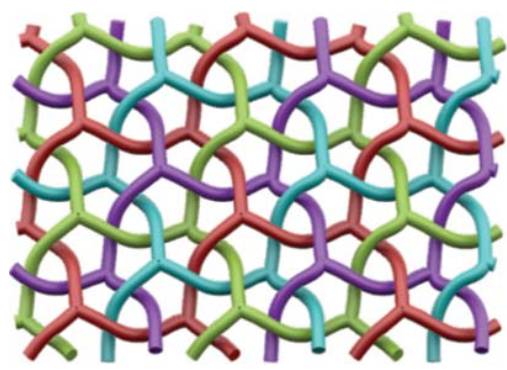

C 2015 International Union of Crystallography

\section{Periodic entanglement III: tangled degree-3 finite and layer net intergrowths from rare forests}

\author{
Myfanwy E. Evans $^{\mathrm{a} *}$ and Stephen T. Hyde ${ }^{\mathrm{b}}$
}

${ }^{\mathbf{a}}$ Institute for Mathematics, Technische Universität Berlin, Germany, and ${ }^{\mathbf{b}}$ Department of Applied Mathematics, Research School of Physics, Australian National University, Australia. *Correspondence e-mail: evans@math.tu-berlin.de

Entanglements of two-dimensional honeycomb nets are constructed from free tilings of the hyperbolic plane $\left(\mathbb{H}^{2}\right)$ on triply periodic minimal surfaces. The 2-periodic nets that comprise the structures are guaranteed by considering regular, rare free tilings in $\mathbb{H}^{2}$. This paper catalogues an array of entanglements that are both beautiful and challenging for current classification techniques, including examples that are realized in metal-organic materials. The compactification of these structures to the genus- 3 torus is considered as a preliminary method for generating entanglements of finite $\theta$-graphs, potentially useful for gaining insight into the entanglement of the periodic structure. This work builds on previous structural enumerations given in Periodic entanglement Parts I and II [Evans et al. (2013). Acta Cryst. A69, 241-261; Evans et al. (2013). Acta Cryst. A69, 262-275].

\section{Introduction}

Thanks to advances in the mathematics of combinatorial tiling theory (Delgado-Friedrichs \& Huson, 1999; DelgadoFriedrichs \& O'Keeffe, 2003, 2005; Chung et al., 1984; Delgado-Friedrichs et al., 2003), systematic enumeration of nets in 3-space is now feasible. A substantial corpus of examples is now available (O'Keeffe et al., 2008; Bonneau \& O'Keeffe, 2015). Most of these examples are characterized by the net topology, and the entanglement of net edges is ignored. The enumeration of multiple catenated nets, or self-entangled nets, is a less developed programme, despite its intrinsic interest and relevance to some aspects of materials. Alexander Wells and Fischer, Koch and Sowa recognized the importance of multiple sphere packings and nets, and discussed some examples in detail (Wells, 1977; Fischer \& Koch, 1976; Koch et al., 2006; Sowa, 2009). They are often encountered in typically highly porous metal-organic frameworks, whose various catenation types have been the subject of a number of studies (Batten \& Robson, 1998; Chen et al., 2001; Blatov et al., 2004, 2014; Reineke et al., 2000; Miller, 2001). They are also found in bicontinuous liquid crystalline mesophases and related mesoporous tricontinuous inorganic derivatives (Han et al., 2009; Kirkensgaard et al., 2014). Novel 'polycontinuous' open foam-like patterns have also been formed starting from multiple nets (Hyde \& Ramsden, 2000; Hyde et al., 2009; Schröder-Turk et al., 2013).

Though we can find no systematic exploration of interwoven nets, the possibility of forming regular patterns from multiple interwoven nets was known, for example, to the Dutch graphic artist M. C. Escher (Schattschneider, 2004). Succeeding mathematical artists have also explored related patterns; see, for example, Rinus Roelofs (Emmer \& Schattschneider, 2003). A classification of entanglement in chemical frameworks is undertaken in Carlucci et al. (2003b, 
2014). This approach gives details of entanglement based on polycatenation, self-catenation and interpenetration of edges through other closed cycles, as well as entanglements of a Borromean nature, involving no interpenetration. New techniques also examine these entanglements from the perspective of 'ideal' energy-minimizing geometry, inspired by the characterization of tight knots (Evans et al., 2015).

A developing approach is to use triply periodic minimal surfaces (TPMSs) as surface scaffolds to transform twodimensional patterns in the hyperbolic plane $\left(\mathbb{H}^{2}\right)$ into threedimensional Euclidean $\left(\mathbb{E}^{3}\right)$ structures with varying topology and entanglement. A variety of structures in $\mathbb{E}^{3}$, from standard nets to filament weavings, are constructed via tilings of TPMSs that are projections of tilings in $\mathbb{H}^{2}$ (Robins et al., 2004b; Ramsden et al., 2009; Hyde et al., 2015; Evans et al., 2013a,b; Castle et al., 2012). Structures have so far been constructed on the genus-3 Primitive $(P)$ and Diamond $(D)$ surfaces described by Schwarz, Schoen's Gyroid $(G)$ surface (Schoen, 1970), and Schwarz's Hexagonal $(H)$ surface (Fig. 1).

Tilings of $\mathbb{H}^{2}$ can be constructed to any level of complexity, using the tools of combinatorial tiling theory (Huson, 1993; Delgado-Friedrichs, 2003), see §2. Many (though far from all) tessellations of $\mathbb{H}^{2}$ have two-dimensional hyperbolic symmetries that are commensurate with those of the hyperbolic TPMSs. Some of those cases have translation subgroups that are common to the translation group of the TPMS; we call them 'crystallographic hyperbolic groups' (Hyde et al., 2014). We can therefore map those hyperbolic tilings onto TPMSs, by aligning the corresponding symmetry elements in the tiling of $\mathbb{H}^{2}$ with those on the TPMS, and form reticulations of the TPMS by tile edges that are periodic in $\mathbb{E}^{3}$ and form crystalline nets, whose unit cell is precisely that of the underlying TPMS. This approach has been used to enumerate specific classes of nets in some detail (Hyde et al., 2015). The details of the technique as well as lists of examples are explained elsewhere (Ramsden et al., 2009; Hyde et al., 2014).

This technique has been extended to include tiling $\mathbb{H}^{2}$ by infinite tiles, called free tilings, whose edge arrays are related to decorations of $\mathbb{H}^{2}$ by trees instead of conventional tessellations (with finite polygons) (Hyde \& Oguey, 2000; Evans et $a l ., 2013 a, b)$. The simplest examples of free tilings are 'regular', with symmetrically equivalent faces, edges and vertices, like the five Platonic polyhedra [and the 'lunar' polyhedra (Castle et al., 2012)], and 'dense'. Two classes of

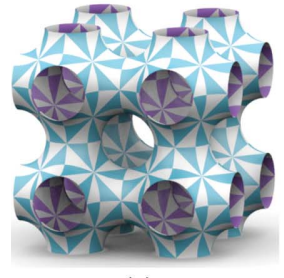

(a)

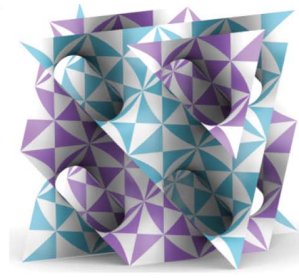

(b)

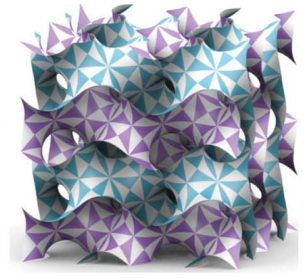

(c)

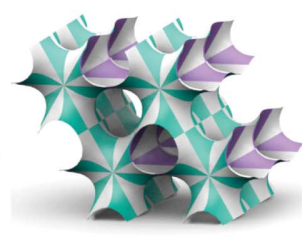

(d)
Figure 1

Genus-3 triply periodic minimal surfaces: $(a)$ Schwarz's primitive $(P)$ surface, $(b)$ Schwarz's diamond $(D)$ surface, $(c)$ Schoen's gyroid $(G)$ surface, $(d)$ Schwarz's hexagonal $(H)$ surface. The $P$, $D$ and $G$ surfaces are decorated with a $\star 246$ tiling, and the $H$ surface with a $\star 2226$ tiling [using Conway's orbifold notation for two-dimensional symmetry groups (Conway \& Huson, 2002)]. regular dense free tilings exist in hyperbolic space, distinguished by their tile morphologies: ribbon tilings and branched ribbon tiles (Evans et al., 2013a,b), that are in oneto-one correspondence with each other. The edges of regular dense ribbon tessellations (and related branched ribbon tilings) of $\mathbb{H}^{2}$ are 'dense forests', consisting of close-packed trees (Hyde \& Oguey, 2000). These examples are readily identified, since the convex hulls of adjacent trees also tessellate $\mathbb{H}^{2}$ without gaps. (Indeed, the convex hulls define the related branched ribbon tiling.)

Free tilings of $\mathbb{H}^{2}$ are interesting, because their projections onto TPMSs generally contain a number of disjoint component nets, mutually catenated, or entangled. Net entanglement is a complex feature, analogous, though richer, to the phenomenon of knotting (Castle et al., 2011; Schröder-Turk et al., 2013; Evans et al., 2013a,b, 2015). Just as all distinct knots are topologically equivalent (to a closed loop, $\mathbb{S}^{1}$, in space), but cannot be interconverted without changing under-over crossings, distinct isotopes of nets are topologically equivalent (i.e. they are isomorphs) but cannot be morphed into each other without 'phantom crossings', where edges pass through each other. Similarly, distinct entanglements or catenations of multiple nets cannot be interconverted without edge crossings.

Free tilings of $\mathbb{H}^{2}$ need not be dense. For example, trees can be symmetrically deleted from a regular dense ribbon tiling so that the pattern remains regular, but adjacent trees are separated by ribbon-shaped domains that are edge-free. Many other reticulations of $\mathbb{H}^{2}$ by trees are possible, characterized by convex hulls that do not themselves tessellate $\mathbb{H}^{2}$, leaving gaps. We call these tilings 'rare', by analogy with rare sphere packings (O'Keeffe, 1991). Rare free tilings of $\mathbb{H}^{2}$ contain tiles that are branched ribbons (Evans et al., 2013a,b). (Note that rarification of dense ribbon tilings necessarily morphs the tiles into branched ribbons.) In this paper we discuss the simplest examples of regular rare free tilings, building on the foundations of free tilings explored in two earlier papers that deal with regular dense examples (Evans et al., 2013a,b). In particular, we adopt a similar naming scheme for rare tilings, labelling distinct tilings via the number of their discrete group $N$ and the edge length in the rare forest, l, e.g. $\star 246_{N R T}(l)$, where RT signifies a Regular Tree structure. Admissible groups are listed in Robins et al. (2004b); we adopt the same numbering scheme for groups as listed there.

Further, we introduce a method of characterization of these structures, which involves compactification of the extended structure to a finite object on the tritorus. One can formally identify, or glue, points on an infinite TPMS that are equivalent by a translation vector of the underlying translation lattice of the periodic surface, giving a finite 'compactified' boundary-free surface patch, that is free of translations. For the TPMSs considered here, that compact patch is a closed surface of genus-3 (a tritorus) (Robins et al., $2004 a, b)$. When the surface reticulation 


\begin{tabular}{l|ccccc} 
Chamber & $\mathrm{s} 0$ & $\mathrm{~s} 1$ & $\mathrm{~s} 2$ & $\mathrm{~m} 01$ & $\mathrm{~m} 12$ \\
\hline $\mathrm{A}$ & $\mathrm{A}$ & $\mathrm{B}$ & $\mathrm{A}$ & 4 & 6 \\
$\mathrm{~B}$ & $\mathrm{~B}$ & $\mathrm{~A}$ & $\mathrm{~B}$ & 4 & 6 \\
\hline
\end{tabular}
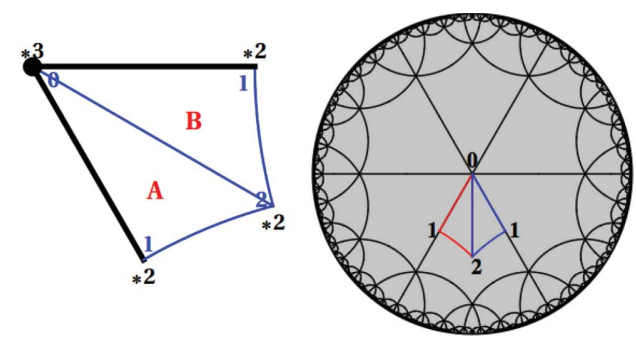

Figure 2

The Delaney-Dress representation of a regular $\{4,6\}$ tiling on the $\star 2223$ orbifold. The edge passes along two mirror boundaries from the $\star 3$ site to two $\star 2$ sites.

\begin{tabular}{l|ccccc} 
Chamber & s0 & s1 & s2 & m01 & m12 \\
\hline A & A & B & A & 4 & 6 \\
B & B & A & B & 4 & 6 \\
\hline
\end{tabular}

Figure 3

The Delaney-Dress representation of a regular ribbon tiling on the $\star 2223$ orbifold. The edge passes along the mirror boundary from the $\star 3$ site to the $\star 2$ site (Evans et al., 2013a).

is maintained through the compactification process, a finite embedded graph results, which is an embedding of the quotient graph of the net (Delgado-Friedrichs \& O'Keeffe, 2005; Eon, 2005). In general, these embeddings are complex and entangled.

\section{Nomenclature}

In the interest of clarity, we first define terms. A graph is a topological object without geometry, defined by points and their mutual connections via edges; simple graphs have no more than one edge between any vertex-pair. We discuss here both finite and infinite graphs, with an (un)bounded number of vertices and edges. An embedding of a graph is a geometric realization of that graph in a space, with assigned geometry (e.g. coordinates) for vertices and all edge points. The number of independent translation vectors of the graph embedding defines its periodicity: we are concerned here with 0 - and 2-periodic graph embeddings. The number of edges that share a vertex characterizes the degree of that vertex; if all vertices have equal 'valence' (e.g. z), the graph and its embeddings have degree $z$. A $k$-connected graph requires at least $k$ vertices and their associated edges to be deleted from the graph to split it into more than a single disjoint graph. In order to classify discrete groups of hyperbolic space, we adopt the orbifold concept, developed by Thurston (1980), which essentially describes all symmetry operations acting on a single asymmetric domain, and use Conway's orbifold nomenclature (Conway, 1992). Here we deal only with the 'Coxeter' and 'stellate' classes, whose orbifolds contain exclusively mirror boundaries and centres of rotational symmetry (cone points), respectively (Hyde et al., 2014). Examples discussed here are derived from Coxeter and stellate tilings belonging to the orbifolds $\star 2^{4} 3$ and $2^{4} 3$ with four distinct twofold centres of rotation and one threefold. ${ }^{\mathbf{1}}$

Tilings of $\mathbb{H}^{2}$ can be constructed of arbitrary complexity, using the tools of Delaney-Dress combinatorial tiling theory. This technique has been explained in detail elsewhere (Huson, 1993; Delgado-Friedrichs, 2003) and we give a very brief summary only here. Delaney-Dress theory encodes tilings via involutions of chambers through edges of three types, $s 0, s 1$ and $s 2$. Chambers are constructed from the tiling by forming a star of edges from the tile mid-points to all mid-points of the tile edges as well as tile vertices. Those edges along with the edges of the underlying tiling form chambers whose vertices lie on tile vertices (labelled as 0 vertices), edges (1) or faces (2). Chamber edges carry labels corresponding to the label of the opposite vertex (not on the edge). Chambers are labelled $A, B, C, \ldots$ and those chambers related by symmetries of the underlying orbifold carry the same label. Adjacent chambers are related by involutions, indexed by their common edge. Those involutions determine the orbifold. The tiling topology is captured by indices $m 01$ and $m 12$ that correspond to the multiplicity of each chamber around a tile centre and vertex, respectively (equal to the indices in the Schläfli symbol). The combined encoding of involutions $s 0, s 1, s 2$ and orders of $m 01$ and $m 12$ (the ' $\mathrm{D}$-symbol') uniquely defines the tiling. The $\mathrm{D}$-symbol is tabulated in Fig. 2 for a tiling with symmetry $\star 2223$.

The corresponding description of free tilings requires a further extension of this nomenclature. We form free tilings by deleting 'ghost' edges from a conventional tiling (with finite faces), and preserve the Delaney-Dress chamber structure of the parent conventional tiling. By associating each free tiling with a conventional tiling, free tilings inherit the enumerable structure of D-symbols. These free tilings are denoted by the original Delaney-Dress encoding with an additional signifier, namely a $\overline{1}$-vertex rather than the standard 1 -vertex, on the chambers that now contain a ghost edge. The symbol may be tabulated identically to regular tilings; however the chambers containing real edges are given in bold font, and the chambers with ghost edges in regular font. The D-symbol is tabulated in Fig. 3 for a free tiling with symmetry $\star 2223$. We note that rigorous description of free tiling theory is still in progress (Evans et al., 2013a).

\footnotetext{
${ }^{1}$ We have modified Conway's orbifold naming scheme. Our $2^{4} 3$ is his 22223 . Our abbreviated form highlights the difference between this orbifold and its close relative $2^{3} 3$ (2223).
} 


\section{Maximal symmetry of rare regular tilings}

In our previous analyses of regular free tilings, we established that regular ribbon degree- $k$ tilings with orbifolds $\star 222 \mathrm{k}$, $2 \star 2 \mathrm{k}, 222 \mathrm{k}$ and $2 \mathrm{k} \times$ form dense patterns in $\mathbb{H}^{2}$ (Evans et al., $2013 a, b)$. The simplest examples are degree-3, whose least symmetric members (with the largest orbifold area) are decorations of the 2223 orbifold [i.e. group 118 in our numbering scheme (Robins et al., 2004b; Robins, 2015)]. (Note that regular ribbon tilings with $23 \times$ symmetry necessarily embed on the $P, D$ and $G$ TPMSs to form reticulations with $2 \star 23$ symmetry.) Regular rare free tilings necessarily have lower symmetry. We find only one symmetry group commensurate with the $P, D$ and $G$ TPMSs that supports such tilings, namely $2^{4} 3$. Additionally, the $H$ TPMS supports two potential symmetry groups, $\star 2{ }^{4} 3$ and $2^{4} 3$.

\section{Embeddling $\star 2^{4} 3$ and $2^{4} 3$ in $\mathbb{H}^{2}$}

Consider free tilings that are regular (1-transitive faces, edges and vertices), where the tile boundaries are composed of vertices and edges and the tiles themselves are internally branched. These tilings have symmetry $\star 2^{4} 3$ or $2^{4} 3$.

The Delaney-Dress representation of the decoration, adapted for free tilings in Evans et al. (2013a), of the $\star 2^{4} 3$ domain is given in Fig. 4. This tiling maps to the $H$ surface via the embedding of the $\star 243$ pattern as a subgroup of the $H$ surface symmetry, namely $\star 2226 / \circ 00$. The tiling (not yet embedded) is labelled by the group-subgroup number as listed in Robins et al. $(2004 a, b)$, in this case $26 R T$ before

\begin{tabular}{l|ccccc} 
Chamber & s0 & s1 & s2 & m01 & m12 \\
\hline A & A & B & A & 8 & 4 \\
B & C & A & B & 8 & 4 \\
C & B & D & C & 8 & 6 \\
D & D & C & D & 8 & 6 \\
\hline
\end{tabular}

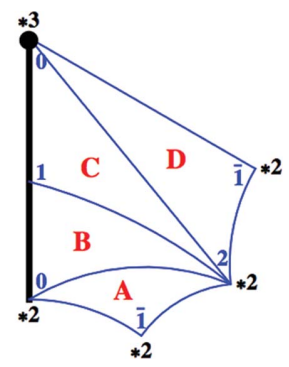

Figure 4

The Delaney-Dress symbol for the $\star 2^{4} 3$ orbifold decoration having one edge passing from the $\star 3$ vertex along the mirror boundary to a $\star 2$ vertex, called $26 R T$. A visual representation of the decoration and chambers on the orbifold is also shown.
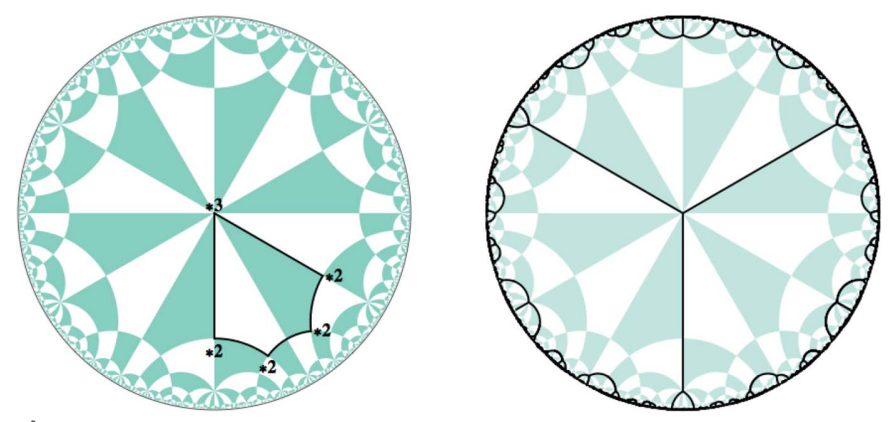

Figure 5

One embedding of the $\star 2^{4} 3$ domain into the $\star 2226$ tiling of $\mathbb{H}^{2}$, and the resulting free tiling: $\star 2226_{26 R T}\left(\cosh ^{-1}(13.9)\right)$. embedding in the $\star 2226 / \circ 0 \circ$ tiling. There is one embedding of the tiling $26 R T$ into the $\star 2226$ tiling that is commensurate with the translational symmetries of the $H$ surface (०००) (Robins $e t$ al., 2004a). This embedded free tiling is shown in Fig. 5, labelled $\star 2226_{26 R T}\left(\cosh ^{-1}(13.9)\right)$, corresponding to the (rare tree) tiling symmetry, the group-subgroup index, and the length in $\mathbb{H}^{2}$ of the tree edges.

The sole example of a regular free tiling of the $2^{4} 3$ orbifold contains a single edge passing from the threefold rotation site to the twofold rotation (labelled $Q_{E}$ in Fig. 6). Since the orbifold is a subgroup of both the $\star 246$ and $\star 2226$ orbifolds, it can be mapped onto the $P, D, G$ surfaces, or the $H$ surface, giving two very distinct group structures. Those structures are determined by the relevant supergroups, namely $\star 2226 / 000$ and $\star 246 / 000$ for the $H$ and $P, D, G$ surfaces, respectively. Accordingly, this tiling is denoted $22 R T$ as a reticulation of the $H$ surface, and $49 R T$ as a reticulation of the $P, D$ and $G$ surfaces.

The D-symbol encodes the topology and two-dimensional symmetry of a tiling, but not its explicit geometry. In general, distinct two-dimensional embeddings are possible, since the orbifold geometry is not unique. In addition, since we are mapping the tilings onto TPMSs embedded in threedimensional Euclidean space and a single orbifold domain can embed in more than one way on the surface, further structural multiplicity is possible. Free tilings on the cubic and hexagonal TPMSs are generated by all possible embeddings of $\star 2^{4} 3$ and $2^{4} 3$ orbifolds as subgroups of $\star 246$ and $\star 2226$, respectively. Admissible orbifold geometries are thus constrained to be commensurate with the parent $(\star 246$ or $\star 2226)$ geometry, so that symmetry sites of the tiling orbifold must coincide with symmetry elements of the parent group of the embedding TPMS.

In contrast to the Coxeter case, stellate orbifolds - which contain rotation centres - have an unlimited number of embeddings within the $\star 246$ or $\star 2226$ setting. The full space of possible embeddings for orbifolds of the form $222 \mathrm{k}$ (for $k>1$ ) is detailed in Evans et al. (2013a). In short, embedding the $222 \mathrm{k}$ orbifolds leads to a two-parameter family of orbifold domains: we may index all possible quadrilateral domains of the $222 \mathrm{k}$ orbifold exactly by embeddings of parallelograms of unit area in $\mathbb{Z} \times \mathbb{Z}$.

The $2^{4} 3$ orbifold is double the size of a 2226 orbifold. An embedded $2^{4} 3$ domain may be constructed by taking a 2226

\begin{tabular}{l|ccccc} 
Chamber & s0 & s1 & s2 & m01 & m12 \\
\hline A & E & B & E & 8 & 4 \\
B & C & A & F & 8 & 4 \\
C & B & D & G & 8 & 6 \\
D & H & C & H & 8 & 6 \\
E & A & F & A & 8 & 4 \\
F & G & E & B & 8 & 4 \\
G & F & H & C & 8 & 6 \\
H & D & G & D & 8 & 6 \\
\hline
\end{tabular}

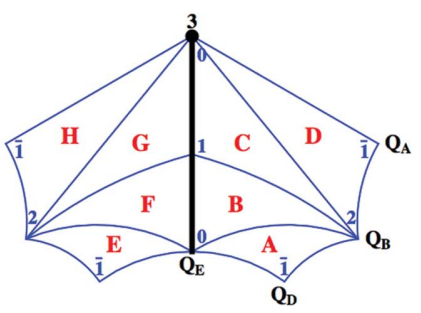

Figure 6

The encoding for the $2^{4} 3$ orbifold decoration having one edge passing from the threefold rotation to the twofold rotation marked $Q_{E}$. This tiling is called $22 R T$ as a member of the $\star 2226 / 000$ quotient group, or $49 R T$ as a member of $\star 246 / 000$. 
symmetry group and doubling across an edge, where the sixfold rotation becomes a threefold rotation, one twofold rotation vanishes, and two copies of the remaining two twofold rotations give the four twofold rotations of $2^{4} 3$. The twofold rotation that vanishes must be carefully chosen, as only one type of the three distinct types of twofold rotations is suitable.

Regular forests constructed in the $2^{4} 3$ orbifold have a single edge passing across the orbifold domain from the threefold to the twofold rotation site. Thus the twofold rotation defining the edge is sufficient to define each forest. It follows that the set of possible edge locations and lengths is identical to that of the 2226 forests described in Evans et al. (2013a), excluding the cases where the edge lies on the twofold site (of $\star 2226$ that is not present in the $2^{4} 3$ orbifold). The first five members of the family of degree- 3 , regular, rare free tilings commensurate with the $\star 2226$ pattern are tabulated in Fig. 7 . The embedding with the shortest edge length $\left(\cosh ^{-1}(13.9)\right)$ has additional symmetry, equivalent to the $\star 2226_{26 R T}\left(\cosh ^{-1}(13.9)\right)$ structure.

We have also deduced the rare, regular, free degree- 3 forests commensurate with the $P, D$ and $G$ surfaces. Here, too, we label these tilings $\star 246_{N R T}(l)$, where $N$ denotes the group
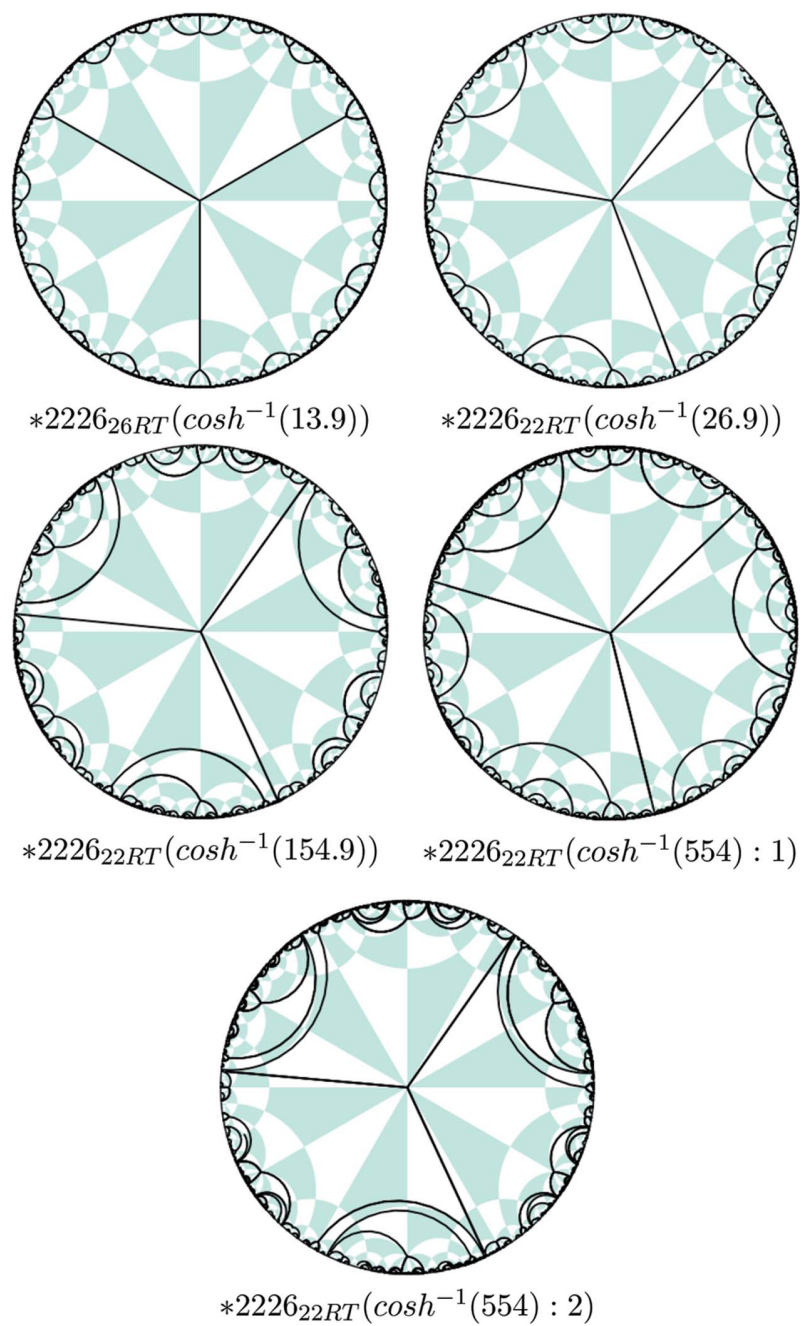

Figure 7

The lowest-order members of the family of degree-3, regular, rare free tilings commensurate with the $\star 2226$ pattern. number in Robins et al. (2004b) and $l$ is the edge length. The first five members of the family of degree-3, regular, rare free tilings commensurate with the $\star 246$ pattern are tabulated in Fig. 8.

\section{Embedding $2^{4} 3$ on the TPMS: entangled nets from regular $2^{4} 3$ patterns}

A surface reticulation inherits three-dimensional symmetries from its two-dimensional orbifold and the underlying surface pair uniquely (Hyde et al., 2014). It turns out that all $2^{4} 3$ tilings in the $P, D$ or $G$ surfaces give rhombohedral three-dimensional Euclidean patterns with space group $R 32$. Similarly, $2^{4} 3$ tilings embedded in the $H$ surface result in patterns with space group P312. Examples of fundamental domains of the $2^{4} 3$ embedding in the extended surfaces are shown in Fig. 9.

By construction, all of our rare tilings have translation subgroups that respect all the translations of the related (oriented) TPMS. Reticulations of the compactified TPMS formed by identifying all translationally equivalent surface sites are quotient graphs of the infinite net on the surface.
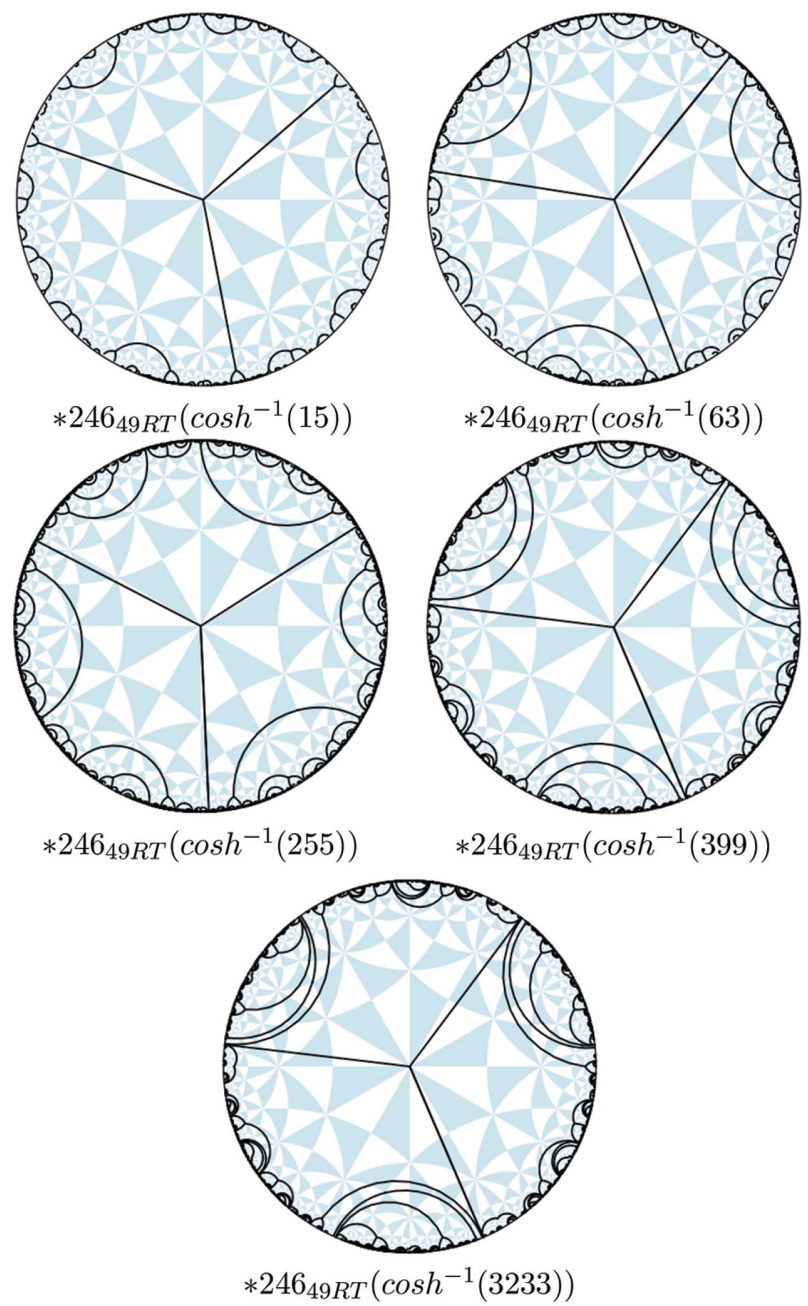

Figure 8

The lowest-order members of the family of degree- 3 , regular, rare free tilings commensurate with the $\star 246$ pattern. 
Table 1

Sparse degree-3 nets.

The nomenclature and net descriptors are explained in the text.

\begin{tabular}{|c|c|c|c|}
\hline Structure & Figure & Topology & Notes \\
\hline$H_{26 R T}\left(\cosh ^{-1}(13.9)\right)$ & Fig. 10 & $\theta$-graph & Untangled finite graphs \\
\hline$H_{22 R T}\left(\cosh ^{-1}(26.9)\right)$ & Fig. 11 & hcb & Two-dimensional Borromean layers \\
\hline$H_{22 R T}\left(\cosh ^{-1}(154.9)\right)$ & Fig. 12 & hcb & Threefold interpenetrated 2-periodic layers, DoC $=6$, Hopf links \\
\hline$H_{22 R T}\left(\cosh ^{-1}(554): 1\right)$ & Fig. 13 & hcb & $\begin{array}{l}\text { Threefold two-dimensional Borromean combined with three-dimensional Borromean, no } \\
\text { interpenetration, IoS }=3\end{array}$ \\
\hline$H_{22 R T}\left(\cosh ^{-1}(554): 2\right)$ & Fig. 14 & $\theta$-graph & Two-dimensional layers of Hopf link catenated $\theta$-graphs \\
\hline$D_{49 R T}\left(\cosh ^{-1}(15)\right)$ & & hcb & Trivial, untangled \\
\hline$D_{49 R T}\left(\cosh ^{-1}(63)\right)$ & Fig. 15 & hcb & Fourfold interpenetrated layers, DoC $=3$ \\
\hline$D_{49 R T}\left(\cosh ^{-1}(399)\right)$ & Fig. 16 & hcb & Single 2-periodic component, 6-cycles are trefoil knots \\
\hline$D_{49 R T}\left(\cosh ^{-1}(3233)\right)$ & Fig. 17 & hcb & Single 2-periodic component, 6 -cycles are $(4,3)$ torus knots \\
\hline$P_{49 R T}^{45 \pi T}\left(\cosh ^{-1}(15)\right)$ & & hcb & Trivial, untangled \\
\hline$P_{49 R T}\left(\cosh ^{-1}(63)\right)$ & & $\theta$-graph & Trivial, untangled \\
\hline$P_{49 R T}\left(\cosh ^{-1}(255)\right)$ & & hcb & Fourfold interpenetrated layers, DoC $=3$, equivalent to $D_{49 R T}\left(\cosh ^{-1}(63)\right)$ \\
\hline$P_{49 R T}\left(\cosh ^{-1}(399)\right)$ & Fig. 18 & hcb & Three-dimensional Borromean structure, $\operatorname{IoS}=1$ \\
\hline$P_{49 R T}\left(\cosh ^{-1}(3233)\right)$ & Fig. 19 & hcb & Catenated three-dimensional structure, $\mathrm{DoC}=12, \mathrm{IoS}=2$ \\
\hline$G_{49 R T}^{+}\left(\cosh ^{-1}(63)\right)$ & Fig. 20 & hcb & Three-dimensional Borromean structure, $\operatorname{IoS}=1$ \\
\hline$G_{49 R T}^{-}\left(\cosh ^{-1}(63)\right)$ & & hcb & Trivial, untangled \\
\hline$G_{49 R T}^{+}\left(\cosh ^{-1}(255)\right)$ & Fig. 21 & hcb & $2 \times$ three-dimensional Borromean structure, $\operatorname{IoS}=2$ \\
\hline$G_{49 R T}^{-}\left(\cosh ^{-1}(255)\right)$ & Fig. 22 & hcb & $\begin{array}{l}\text { Two-dimensional to three-dimensional catenated structure. Catenation same as the two- } \\
\text { dimensional to two-dimensional interpenetration in } H_{22 R T}\left(\cosh ^{-1}(154.9)\right) \text {. DoC }=6 \text {, IoS }=1\end{array}$ \\
\hline$G_{49 R T}^{+}\left(\cosh ^{-1}(399)\right)$ & Fig. 23 & hcb & Catenated three-dimensional structure equivalent to $P_{49 R T}\left(\cosh ^{-1}(3233)\right) . \mathrm{DoC}=12, \operatorname{IoS}=2$ \\
\hline
\end{tabular}

Since the compactified TPMSs are genus-3 (or $\circ 00$ ) for the $P$, $D, G$ and $H$ surfaces, the corresponding quotient graphs are of degree-3 reticulating a genus-3 manifold. Since the parent net is regular, all vertices in the quotient graph are interchangeable via graph automorphisms. The rare tilings have a vertex at the threefold site on the $2^{4} 3$ orbifold (with $\frac{1}{3}$ of a vertex per orbifold copy). Six copies of the decorated $2^{4} 3$ orbifold tile a single (genus-3) $0 \circ \circ$ orbifold, giving two vertices per genus-3 manifold and the corresponding quotient graph that is formed from the infinite reticulation modulo the set of translation vectors of the (oriented) TPMS (excluding those translations that swap sides of the surface). The underlying regular degree3 net therefore has exactly two vertices per unit cell. This implies that the quotient graph of the reticulated patterns on all four TPMSs is a $\theta$-graph (two vertices connected by three edges, $K_{2}^{3}$ ). Since the graph vertices are located at sites with threefold rotational symmetry (in both the two-dimensional orbifold and three-dimensional space-group settings), the resulting nets formed by these reticulations will be either

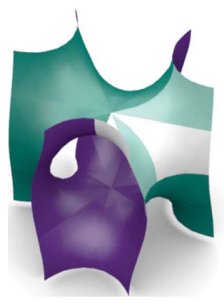

(a)

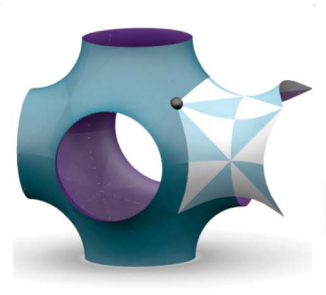

(b)

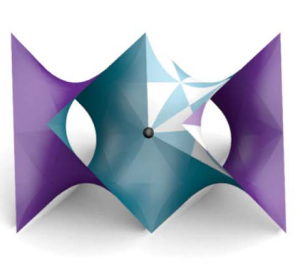

(c)

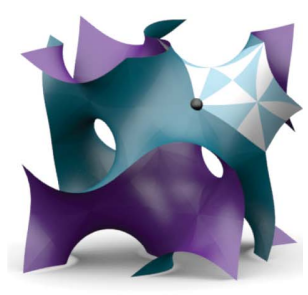

(d)
Figure 9

(a) The highlighted tile shows one possible $2^{4} 3$ fundamental domain within the $H$ surface, itself coloured by tiles that describe the $\star 2226$ domains. This tile builds up to the complete surface via the $P 312$ space-group operations. (b), (c), (d) A highlighted tile of the $2^{4} 3$ symmetry group within the $P$, $D$ and $G$ surfaces, respectively. This curved tile gives the complete surface through the $R 32$ space group. arrays of discrete $\theta$-graphs, or arrays of (translationally equivalent) hcb nets (O'Keeffe et al., 2008). Further, less symmetric regular examples can also have no more than two vertices per unit cell if they reticulate any genus-3 TPMS and respect all translations, since the number of copies of their orbifolds within 000 cannot exceed six. So no degree-3 weaving that forms from a regular rare tiling on a genus-3 TPMS can contain 3-periodic component nets. It is noteworthy that srs nets, whose intergrowths are common among regular dense tilings, are suppressed for these maximally symmetric regular rare tilings. (We note that other arrangements are possible if we choose irregular tilings, thereby breaking the threefold symmetry of the pattern.)

Consider further other regular free tilings that are potentially compatible with genus-3 TPMSs, namely those with degrees $k=4,6$. In those cases, regular rare tilings are incompatible with the crystallographic hyperbolic groups on the $P, D, G$ and $H$ surfaces that contain all translations of the (oriented) genus-3 surfaces. However, crystalline nets (of degrees 3 as well as 4 and 6) can be formed from rare free tilings on these TPMSs if we allow patterns whose orbifolds lie outside our restricted class of genus-3 crystallographic hyperbolic groups.

A summary of the structures that result from the reticulation of these tilings over each of the $H, D, P$ and $G$ surfaces is given in Table 1 . The structures are named to reflect the original tiling in $\mathbb{H}^{2}$ as well as the surface over which the tilings were reticulated: $P_{49 R T}\left(\cosh ^{-1}(15)\right)$ is the reticulation of the $\star 246_{49 R T}\left(\cosh ^{-1}(15)\right)$ tiling over the 
$P$ surface. Some details of the resulting three-dimensional structure are given, such as the Index of Separation (IoS), which ascertains how many components must be removed for the structure to separate into two (Carlucci et al., 2003b), and the Density of Catenation (DoC), which is a measure of catenation in a structure: it collates the number of distinct cycles with which a single hexagon is threaded (Carlucci et al., $2003 \mathrm{~b}$ ). We also use the description Borromean to denote structures whose cycles are entangled in a Borromean ring motif (Carlucci et al., 2003a). Note that this is similar to, but formally inequivalent to, Brunnian entanglements, since the removal of one component of a Borromean entangled structure in some cases will cause the structure to separate into two
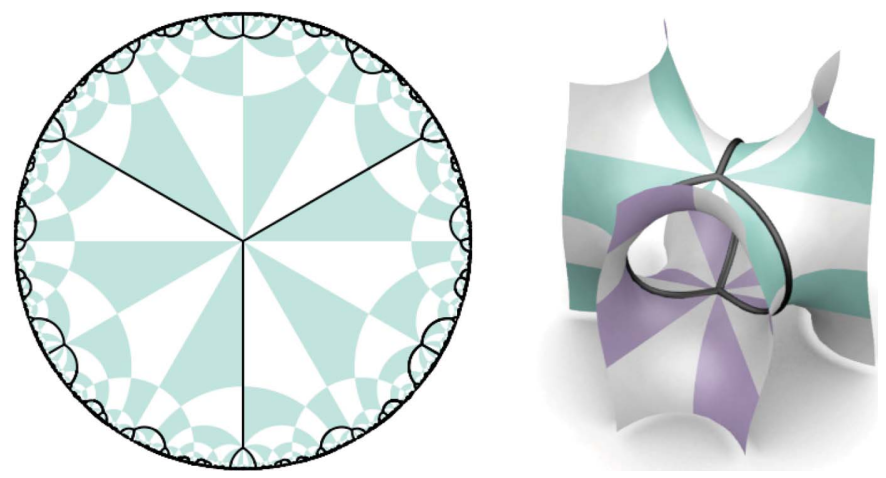

Figure 10

(Left) The $\star 2226_{26 R T}\left(\cosh ^{-1}(13.9)\right)$ in $\mathbb{H}^{2}$ and (Right) the $H_{26 R T}\left(\cosh ^{-1}(13.9)\right)$ structure shown on the $H$ surface, which consists of untangled finite $\theta$-graphs.

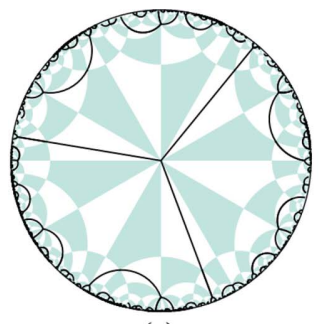

(a)

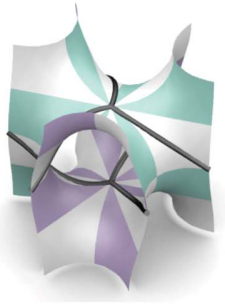

(b)

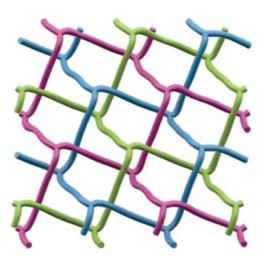

(c)
Figure 11

(a) The $\star 2226_{22 R T}\left(\cosh ^{-1}(26.9)\right)$ rare free tiling in $\mathbb{H}^{2} ; \quad$ (b) $H_{22 R T}\left(\cosh ^{-1}(26.9)\right)$ on the $H$ surface and $(c)$ in $\mathbb{E}^{3}$. The resulting pattern is a parallel stack of disjoint layers, each containing a trio of hcb nets forming a two-dimensional Borromean entangled pattern.

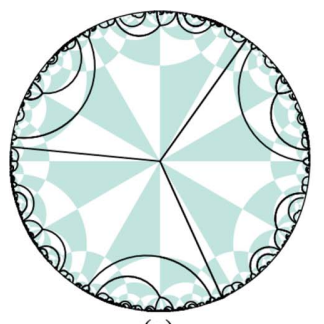

(a)

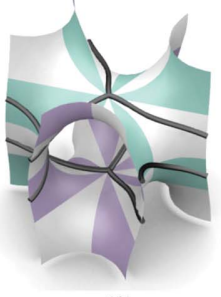

(b)

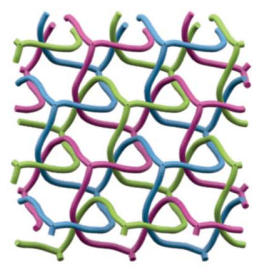

(c)
Figure 12

(a) The $\star 2226_{22 R T}\left(\cosh ^{-1}(154.9)\right) \quad$ tiling in $\mathbb{H}^{2}$. (b) The $H_{22 R T}\left(\cosh ^{-1}(154.9)\right)$ structure on the $H$ surface, resulting in (c) a repeated, parallel set of 2-periodic interpenetrating layers, each containing three hcb nets. $\mathrm{DoC}=6$. parts, not separate into individual components as required by Brunnian conditions. Data files (cgd) for these structures are available as supporting information.

The patterns formed on the $H$ surface with the shortest edges are spatially simple structures. Examples include trivial entanglements, such as the array of disjoint untangled $\theta$-graphs $\left(H_{26 R T}\left(\cosh ^{-1}(13.9)\right)\right.$ (Fig. 10).

Additional reticulations of the $H$ surface yield a number of subtle entanglements of multiple hob nets. The $H_{22 R T}\left(\cosh ^{-1}(26.9)\right)$ example leads to a stack of parallel layers, each containing a trio of hcb nets, which are mutually entangled within each layer (Fig. 11). Adjacent layers are however not entangled with each other. Within each layer, the nets entangle such that hexagonal rings of each component net form Borromean links: removal of one hcb net leaves the remaining pair of nets unentangled. Thus, in this case, the IoS is 1. Its reticulation on the $H$ surface has space-group symmetry P312; the pattern can be further symmetrized without altering its entangled structure by straightening all edges, giving a pattern with symmetry $P \overline{3} 1 \mathrm{~m}$. The twodimensional Borromean entanglement has been described in the context of chemistry (Carlucci et al., 2003a); a comprehensive account of the occurrence of such structures in metalorganic frameworks can be found in Carlucci et al. (2014).

The $H_{22 R T}\left(\cosh ^{-1}(154.9)\right)$ structure (Fig. 12) is a repeated, parallel set of interpenetrating layers each with three components. It has a $\mathrm{DoC}=6$, where the catenation of pairs of hexagonal rings from distinct hcb nets is via Hopf links, and six edges thread each hexagonal ring (three from each of the remaining two distinct hcb nets).

The $H_{22 R T}\left(\cosh ^{-1}(554): 1\right)$ (Fig. 13) pattern demonstrates the further complexity that can be realized by entangled structures. Like $H_{22 R T}\left(\cosh ^{-1}(26.9)\right)$, it contains stacks of three hcb nets, mutually tangled in a Borromean fashion, without threading of any pair of nets. In contrast to the previous

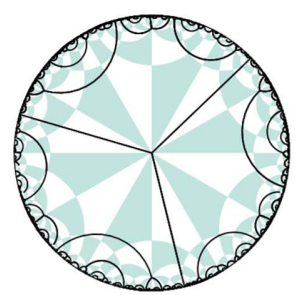

(a)

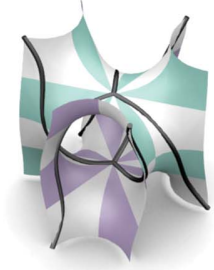

(b)

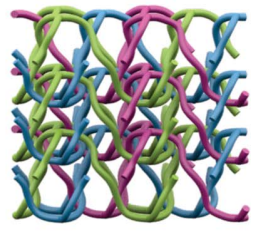

(c)

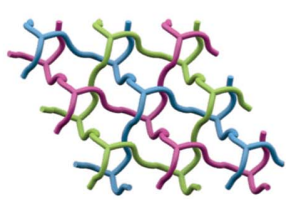

(d)

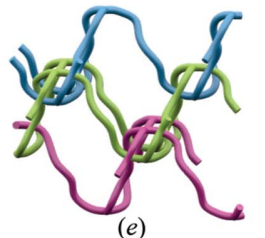

(e)
Figure 13

(a) $\star 2226_{22 R T}\left(\cosh ^{-1}(554): 1\right)$ in $\mathbb{H}^{2}$. (b), (c) $H_{22 R T}\left(\cosh ^{-1}(554): 1\right)$ contains two distinct modes of entanglement, both with Borromean links of cycles, and free of catenation of hexagonal rings from distinct hcb net pairs. $(d)$ One layer of the pattern, revealing the Borromean nature of a trio of hcb nets within a single two-dimensional layer. (e) A single component net from each of three distinct two-dimensional layers, which together mutually entangle, also in Borromean fashion. $\operatorname{IoS}=3$. 
example, however, the layers are themselves also entangled in a Borromean manner. Each hcb component of each twodimensional Borromean layer entangles with components of adjacent layers via a three-dimensional Borromean entanglement (see Carlucci et al., 2003b, 2014). Equivalently, each hcb component within a single copy of the three-dimensional Borromean entanglement has two additional hcb components associated by a two-dimensional Borromean entanglement. The IoS is 3, where an entire layer of two-dimensional Borromean must be removed to separate the structure in two.

The catenation of finite $\theta$-graphs into a 2-periodic array is also possible. The $H_{22 R T}\left(\cosh ^{-1}(554): 2\right)$ reticulation, where the $\theta$-graphs link to their neighbours via a Hopf link, is shown in Fig. 14.

Degree-3 regular rare free tilings on the $D$ surface result in a number of fascinating patterns, including four-component weavings and self-knotted nets. The $D_{49 R T}\left(\cosh ^{-1}(63)\right)$ structure contains four distinct hcb nets (Fig. 15) in a fourfold

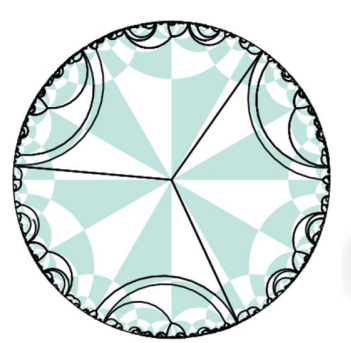

(a)

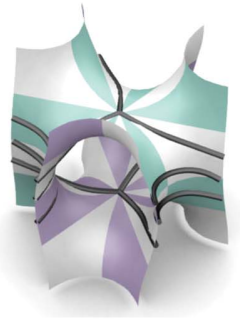

(b)

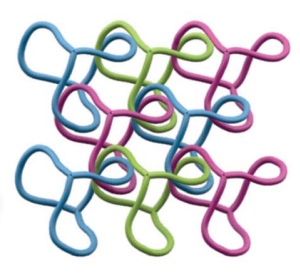

(c)
Figure 14

(a) $\star 2226_{22 R T}\left(\cosh ^{-1}(554): 2\right)$ in $\mathbb{H}^{2}$. (b) The $H_{22 R T}\left(\cosh ^{-1}(554): 2\right)$ reticulation of the $H$ surface, forming arrays of $\theta$-graphs. (c) The resulting pattern in $\mathbb{E}^{3}$ : two-dimensional layers of Hopf link catenated $\theta$-graphs.

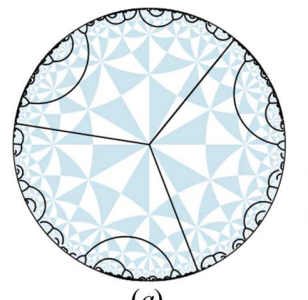

(a)

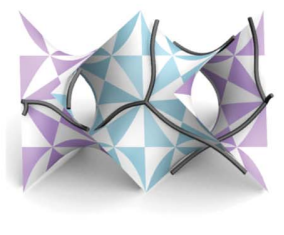

(b)

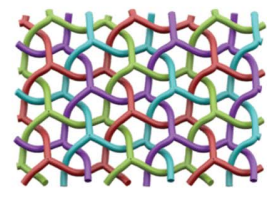

(c)
Figure 15

(a) The $\star 246_{49 R T}\left(\cosh ^{-1}(63)\right)$ tiling in $\mathbb{H}^{2}$. (b), (c) The $D_{49 R T}\left(\cosh ^{-1}(63)\right)$ structure is comprised of disjoint layers with fourfold interpenetration. $\mathrm{DoC}=3$.

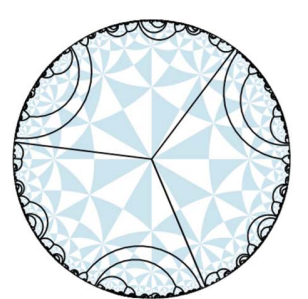

(a)

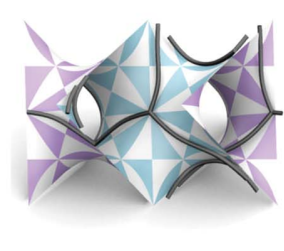

(b)

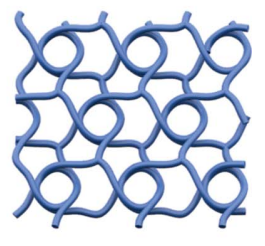

(c)
Figure 16

(a) The $\star 246_{49 R T}\left(\cosh ^{-1}(399)\right)$ tiling in $\mathbb{H}^{2}$, and $(b) D_{49 R T}\left(\cosh ^{-1}(399)\right)$ on the $D$ surface. (c) The resulting pattern in $\mathbb{E}^{3}$ is comprised of disjoint, isolated layers with a single, self-catenated hcb net whose cycles are trefoil knots. interpenetrating pattern. Hexagonal rings belonging to pairs of distinct nets thread each other to form Hopf links, and the DoC $=3$. Carlucci et al. (2014) report four chemical frameworks with fourfold interpenetrating hcb nets: none are equivalent to the example shown here. This pattern is closely related to a beautiful weaving sketched by M. C. Escher, containing a pair of inter-grown hcb nets (Schattschneider, 2004). Where each component (trigonally distorted) hcb net in our weaving has $\overline{3}$ symmetry at the centre of each hexagonal cycle, Escher's (rectangular) nets contain hexagons with twofold symmetry only. The weaving of the two nets is subtly distinct from that of a pair of nets in our structure.

The $D_{49 R T}\left(\cosh ^{-1}(399)\right)$ tiling results in a stack of parallel single self-entangled hcb nets. Individual hexagonal rings form trefoil knots (Fig. 16). In contrast, the $D_{49 R T}\left(\cosh ^{-1}(3233)\right)$ tiling also gives a self-entangled arrangement in $\mathbb{E}^{3}$, whose individual hexagonal rings form $(4,3)$ torus knots (Fig. 17).

The structures that result by mapping these regular, rare, free tilings onto the $P$ surface are also complex. The $P_{49 R T}\left(\cosh ^{-1}(399)\right)$ pattern leads to an array of parallel hcb nets, mutually entangled to form a three-dimensional Borromean structure (Fig. 18). Despite its apparent complexity in Euclidean space, this structure too has been synthesized chemically; a comprehensive account of the occurrence of such structures in metal-organic frameworks can be found in Carlucci et al. (2014).

The $P_{49 R T}\left(\cosh ^{-1}(3233)\right)$ structure is comprised of hcb twodimensional nets which mutually catenate to form a threedimensional structure (Fig. 19). The IoS is 2, which indicates the structure is a twofold structure where the layers are catenated, i.e. if every second net is deleted the structure remains catenated. The full pattern is comprised of two identical copies of this, also mutually interpenetrating.

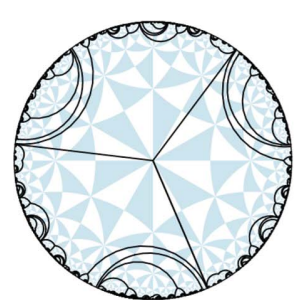

(a)

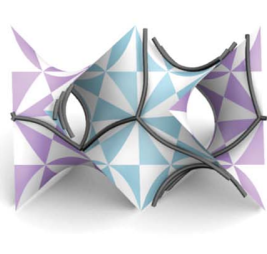

(b)

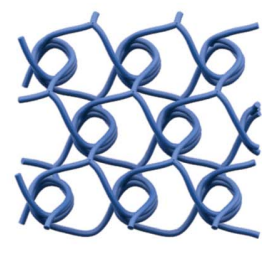

(c)
Figure 17

(a) The $\star 246_{49 R T}\left(\cosh ^{-1}(3233)\right)$ tiling in $\mathbb{H}^{2} . \quad(b), \quad(c)$ The $D_{49 R T}\left(\cosh ^{-1}(3233)\right)$ structure is comprised of disjoint, isolated layers with a single, self-catenated hob net whose cycles are $(4,3)$ torus knots.

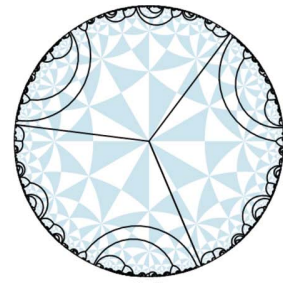

(a) (b)

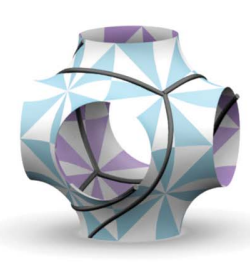

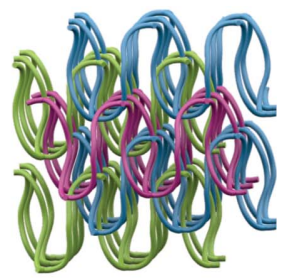

(c)
Figure 18

(a) $\star 246_{49 R T}\left(\cosh ^{-1}(399)\right)$, shown in $\mathbb{H}^{2}$. (b), (c) The entangled net $P_{49 R T}\left(\cosh ^{-1}(399)\right)$, shown on one unit cell of the $P$ surface and in $\mathbb{E}^{3}$, is a three-dimensional Borromean entanglement of hcb components. IoS $=1$. 
In contrast to reticulations of the other TPMSs analysed to date, the lack of two-dimensional reflections in these rare free tilings leads to a pair of patterns for each tiling on the $G$ surface, as discussed in detail elsewhere (Robins et al., 2005). (We denote these distinct patterns ' $G^{+}$' and ' $G^{-}$.) A range of patterns emerges (Figs. 20, 21, 22 and 23), some of which are equivalent entanglements (or isotopes) to previous examples.

\section{Embedded $\theta$-graphs on the tritorus}

The catalogue of examples given in the previous section, with a wealth of entangled structures, is made up of just two component nets: hcb or the $\theta$-graph. Here we explore a little further possible entanglements of the finite $\theta$-graph, which can be generated readily using the apparatus introduced above.

The $P, D$ and $G$ and $H$ surfaces can be considered as partial compactifications of $\mathbb{H}^{2}$, 'rolling' the infinite hyperbolic plane $\left(\mathbb{H}^{2}\right)$ up, to form a surface with three translations, just as an infinite planar pattern in $\mathbb{E}^{2}$ can be rolled into a 1-periodic pattern on the cylinder. Periodic cylindrical patterns can be (abstractly) compactified once more via identification of all points related by the single remaining lattice vector, to give a

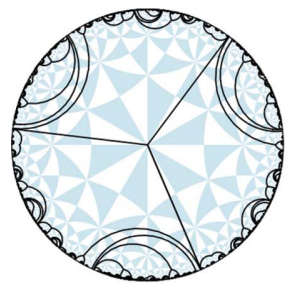

(a)

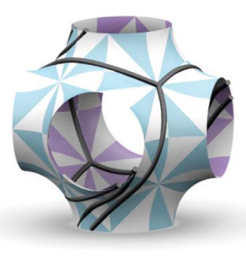

(b)

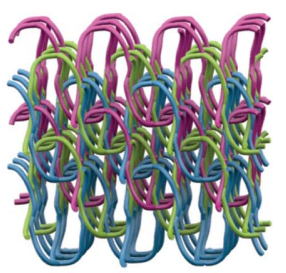

(c)

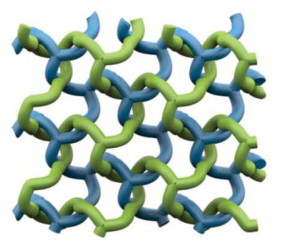

(d)

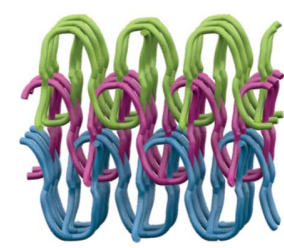

(e)
Figure 19

(a) The $\star 246_{49 R T}\left(\cosh ^{-1}(3233)\right)$ tiling in $\mathbb{H}^{2}$. (b), (c) The structure $P_{49 R T}\left(\cosh ^{-1}(3233)\right)$ is a catenated three-dimensional array of hcb nets. ( $d$ ) Two adjacent catenated layers are shown. (e) A subset of the pattern containing every second layer of the twofold structure, which remains catenated. Here, the DoC $=12$ and the $\mathrm{IoS}=2$.

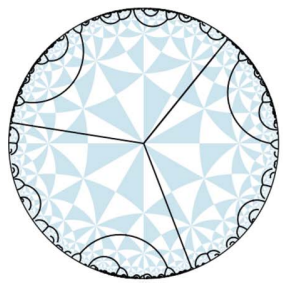

(a)

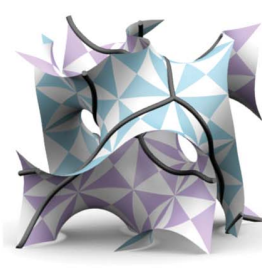

(b)

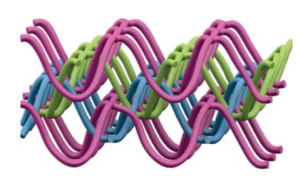

(c)
Figure 20

(a) The $\star 246_{49 R T}\left(\cosh ^{-1}(63)\right)$ tiling of $\mathbb{H}^{2}$. (b) The $G_{49 R T}^{+}\left(\cosh ^{-1}(63)\right)$ reticulation of the $G$ surface, and $(c)$ the structure in $\mathbb{E}^{3}$, a standard threedimensional Borromean entanglement of hcb components, equivalent to the $P_{49 R T}\left(\cosh ^{-1}(399)\right)$ structure. IoS $=1$. (0-periodic) pattern on the (genus-1) torus. Likewise, TPMSs can be further compactified via the remaining three lattice vectors of the surface, forming the genus- 3 tritorus (the orbifold $\circ \circ \circ$ ). The $H$ surface is the partial compactification of $\mathbb{H}^{2}$ towards a tritorus with $\star 2226$ symmetry, and the $P, D$ and $G$ surfaces are related to a $\star 246$ tritorus. Details of the map from $\mathbb{H}^{2}$ to the tritorus for these surfaces are given in Robins $e t$ al. $(2004 a, b)$.

Alternatively, hyperbolic patterns whose symmetry is a subgroup of $\star 2226 / \star 246$ and a supergroup of 000 will seamlessly reticulate the tritorus. Rare, regular hyperbolic forests with symmetry $2^{4} 3$ fulfil this condition, and they will map onto the tritorus to give various embeddings of the $\theta$-graph, stemming from the presence of two regular degree- 3 vertices per tritorus (০০০) domain in $\mathbb{H}^{2}$.

Distinct forests within the same conjugacy class form equivalent reticulations of TPMSs, since the two-dimensional conjugacies of the underlying hyperbolic tiling are also threedimensional conjugacies (corresponding to isometries) on the TPMS. However, these two-dimensional conjugacies are not conjugacies of the tritorus. Therefore a single conjugacy class of hyperbolic forests can result in a number of distinct isotopes of the embedded $\theta$-graph on the tritorus.

These issues are most readily comprehended with reference to the $P$ surface. Compactification of a single unit cell of the $P$ surface gives a tritorus with $\star 246$ symmetry. The compactification process takes a single node from the surface and glues opposite arms, giving a three-looped tritorus. Hyperbolic patterns with symmetry $2^{4} 3$ on this tritorus give two distinct embedded graphs, since one conjugacy of $\mathbb{H}^{2}$ does not correspond to a conjugacy of the $\star 246$ tritorus. Those cases are formed by compactifying two distinct unit cells of the surface, giving two embedded $\theta$-graphs (Fig. 24). One of those choices of unit cell leads to a simpler finite graph: the unit cell is chosen where the reticulation has the least number of connected parts, as illustrated in Fig. 24.

Regular rare forests arising from $2^{4} 3$ tilings related to the $H$ surface $(\star 2226)$ lead to two distinct embedded $\theta$-graphs on the tritorus, since there is one conjugacy of $\mathbb{H}^{2}$ that does not correspond to a conjugacy of this tritorus. We outline the resulting $\theta$-graph embeddings in Table 2, including the related $H$ surface reticulations.

These examples lead to complex entanglements of the $\theta$-graph in addition to the familiar ('trivial') untangled

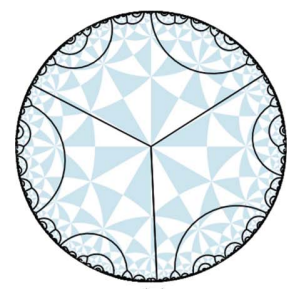

(a)

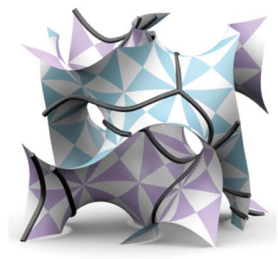

(b)

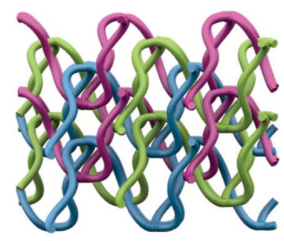

(c)
Figure 21

(a) The $\star 246_{49 R T}\left(\cosh ^{-1}(255)\right)$ tiling in $\mathbb{H}^{2}$. (b) A single unit cell of the $G$ surface showing the structure $G_{49 R T}^{+}\left(\cosh ^{-1}(255)\right)$. (c) The resulting structure in $\mathbb{E}^{3}$ contains two copies of a three-dimensional Borromean entanglement of hcb components sitting one inside the other. $\operatorname{IoS}=2$. 
Table 2

$\theta$-graph embeddings from regular, rare degree- 3 forests.

\begin{tabular}{|c|c|c|c|c|}
\hline Structure & $H$ net & Tritorus $A$ & Tritorus $B$ & Figure \\
\hline$\star 2226_{26 R T}\left(\cosh ^{-1}(13.9)\right)$ & Zero-dimensional $\theta$-graph, isolated & Trivial & Trivial & \\
\hline$\star 2226_{22 R T}\left(\cosh ^{-1}(26.9)\right)$ & Two-dimensional Borromean & Trivial & 3-ravel & Fig. 25 \\
\hline$\star 2226_{22 R T}\left(\cosh ^{-1}(154.9)\right)$ & Interpenetrating hcb, $\mathrm{DoC}=6$, Hopf links only & 3-ravel & Cycles are 3-twist knots & Fig. 26 \\
\hline$\star 2226_{22 R T}\left(\cosh ^{-1}(554): 2\right)$ & $\begin{array}{l}\text { Zero-dimensional to two-dimensional catenation of } \theta \text {-graphs } \\
\text { by Hopf links }\end{array}$ & Cycles are 5-twist knots & Complex entanglement & Fig. 27 \\
\hline
\end{tabular}

embedding. In particular, the $\star 2226_{22 R T}\left(\cosh ^{-1}(26.9)\right)$ and $\star 2226_{22 R T}\left(\cosh ^{-1}(154.9)\right)$ tilings induce a symmetric 3 -ravel on the tritorus, discussed in more detail elsewhere (Castle et al., 2008) (Figs. 25 and 26). This isotope is noteworthy, since it is clearly entangled, yet contains no knots or links, since all cycles in the graph are unknotted and free of links. A ravelled metal-organic molecule has been synthesized, confirming the relevance of these patterns to material structures ( $\mathrm{Li}$ et al., 2011). In contrast, knotted embeddings are generated from the second embedding of $\star 2226_{22 R T}\left(\cosh ^{-1}(154.9)\right)$ tiling and both embeddings of $\star 2226_{22 R T}\left(\cosh ^{-1}(554): 2\right)$ tiling (Figs. 26 and 27).

It is of interest to compare these entanglements with the related hcb arrays formed on the parent $H$ surface by these tilings. In some, though not all, cases, the $\theta$-graph embeddings capture a qualitative sense of the entanglement of the related array of nets on the $H$ surface. For example, the trivial embedding of a $\theta$-graph lifts to an array of disjoint $\theta$-graphs obtained on the $H$ surface. In contrast, the 3-ravel embedding of the $\theta$-graph lifts to the two-dimensional Borromean

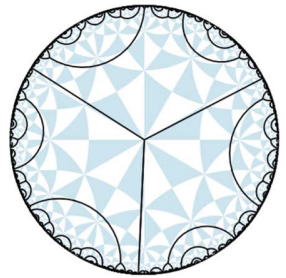

(a)

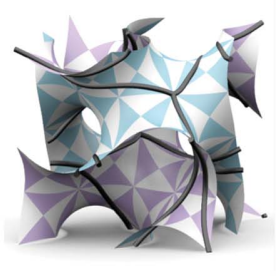

(b)

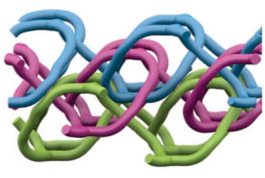

(c)
Table 3

$\theta$-graph embeddings from regular, rare degree- 3 forests.

\begin{tabular}{|c|c|c|c|}
\hline $\mathbb{H}^{2}$ tiling & $\mathbb{E}^{3}$ net & Tritorus & Figure \\
\hline$\star 246_{49 R T}\left(\cosh ^{-1}(15)\right)$ & Trivial hcb nets & Trivial $\theta$-graph & \\
\hline$\star 246_{49 R T}\left(\cosh ^{-1}(63)\right)$ & $\theta$-graphs, isolated & Trivial $\theta$-graph & \\
\hline$\star 246_{49 R T}\left(\cosh ^{-1}(255)\right)$ & $\begin{array}{l}\text { Fourfold interpenetrating } \\
\text { hcb nets }\end{array}$ & 3 -ravel & \\
\hline$\star 246_{49 R T}\left(\cosh ^{-1}(399)\right)$ & $\begin{array}{l}\text { Three-dimensional } \\
\text { Borromean }\end{array}$ & 3-ravel & Fig. 28 \\
\hline$\star 246_{49 R T}\left(\cosh ^{-1}(3233)\right)$ & Catenated twofold & $\begin{array}{l}\text { Cycles are } \\
\text { 3-twist knots }\end{array}$ & Fig. 29 \\
\hline
\end{tabular}

entanglement (Figs. 25 and 11); in both cases the embeddings are free of catenated rings. Further, the highly catenated and entangled infinite frameworks on the $H$ surface are related to more complex embeddings of the $\theta$-graph, containing knotted cycles (Figs. 27 and 14).

We give just a few examples of $\theta$-graph embeddings resulting from the $\star 246$ free tilings, to demonstrate the general nature of this construction. We choose to compactify one unit cell of the $P$ surface reticulation in the orientation given in Fig. 24(a). The resulting embeddings are listed in Table 3.

\section{Discussion}

In closing, we put this work in a broader context. First, the reader may wonder why we have focused on regular forests, consisting of maximally symmetric (regular) arrays of trees in hyperbolic space. There are simpler symmetric tilings of the hyperbolic plane, namely examples whose tiles are finite. We are slowly enumerating those examples too, and that underlies the Epinet project (http://epinet.anu.edu.au), described in detail elsewhere (Ramsden et al., 2009).

Given the unfamiliarity of hyperbolic geometry, it is perhaps illuminating to consider the analogous problem in twodimensional Euclidean space. There, tilings of the plane by regular trees are only possible if the trees are of degree-2, forming simple arrays of lines in the plane. In that case regular examples

(a) The $\star 246_{49 R T}\left(\cosh ^{-1}(399)\right)$ tiling of $\mathbb{H}^{2}$. (b)-(d) The $G_{49 R T}^{+}\left(\cosh ^{-1}(399)\right)$ tiling leads to a threedimensional catenated structure, with equivalent entanglement to the $P_{49 R T}\left(\cosh ^{-1}(3233)\right)$ pattern seen in Fig. 19. Here, the DoC $=12$ and the $\mathrm{IoS}=2$. 


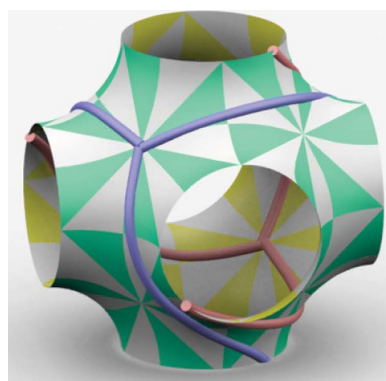

(a)

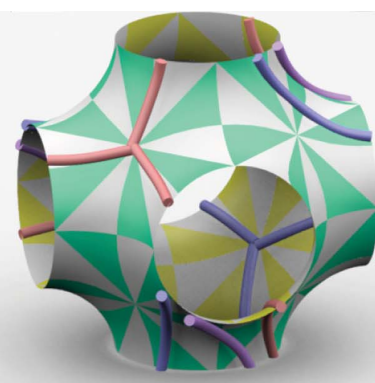

(b)
Figure 24

(a) The decoration of a hyperbolic forest of symmetry $2^{4} 3$ on a unit cell of the $P$ surface. (b) A distinct reticulation of the unit cell, due to an equivalent hyperbolic forest on the $P$ surface. This pattern results by a simple reflection in an edge of the $\star 246$ orbifold. While this operation is both two- and three-dimensional conjugacies of the pattern, it is not a conjugacy of the pattern on the tritorus.

consist of parallel lines. (It is the vastly enhanced scope of parallelism in hyperbolic space that admits the more complex examples in hyperbolic space.) Evidently, in Euclidean space, the concepts of dense and rare packings of parallel lines are

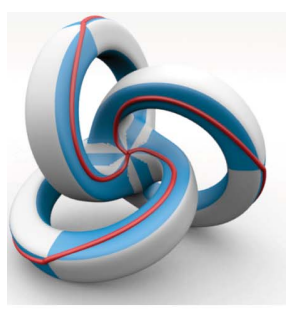

(a)

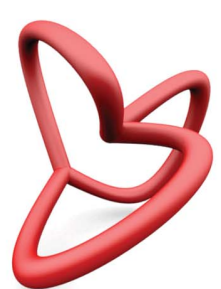

(b)

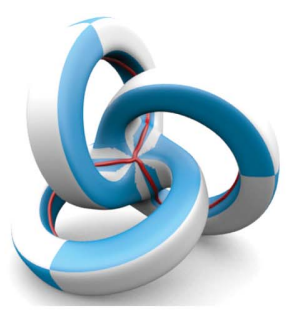

(c)

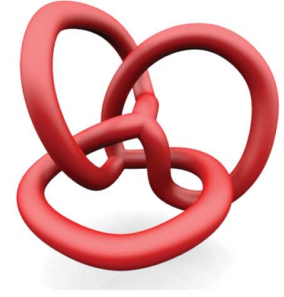

(d)
Figure 25

$(a) \star 2226_{22 R T}\left(\cosh ^{-1}(26.9)\right)$ on the tritorus by one orientation. (b) The resulting embedded graph is a trivial $\theta$-graph. (c) A second orientation, being a conjugacy of the forest in $\mathbb{H}^{2}$ but not on the tritorus, reticulated over the tritorus. (d) This gives a simple 3-ravel (Castle et al., 2008) embedding of a finite $\theta$-graph.
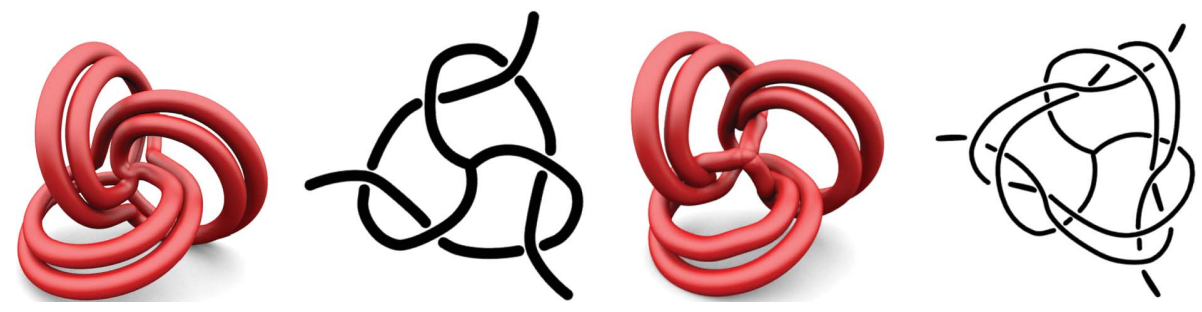

Figure 26

Two orientations of the embedded graph of $\star 2226_{22 R T}\left(\cosh ^{-1}(154.9)\right)$ on the tritorus. The graphs with the second vertex cut for visual simplicity: the first is a simple 3-ravel, the second having knotted cycles.
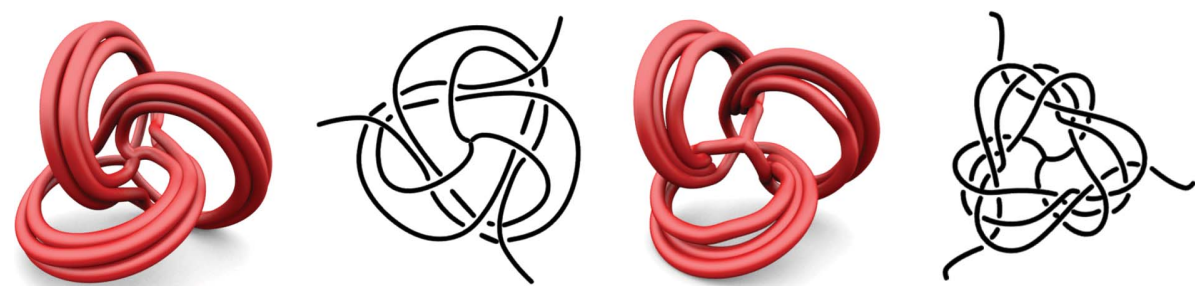

Figure 27

The two graphs from the tritorus reticulation of the forest $\star 2226_{22 R T}\left(\cosh ^{-1}(554): 2\right)$. A representation of these embedded graphs, both having knotted cycles. also trivial: a dense packing is a stack of parallel lines, all rlapping, while rare regular examples are 1-periodic arrays displaced and parallel lines. As discussed above, any cylinder. Thus, regur correspond to multiple parallel disjoint loops or nested helices on the cylinder, respectively.

As we have demonstrated, the wealth of analogous patterns in hyperbolic space is far richer. An infinite variety of hyperbolic forests can be constructed, with trees of arbitrary degree ia judicious tuning of the tree edge lengths. Among those, with symmetrically equivalent vertices and polyhedra, whose edges form regular reticulations on the sphere). And a subset of those forests, whose symmetries correspond to crystallographic hyperbolic groups, map into structures. We have demonstrated that those structures can consist of finite or extended periodic nets. A previous publication analysed the variety of entangled structures that emerge from dense forests on the $P, D$ and $G$ and $H$ surfaces (Evans et al., 2013a). There, forests of degrees 3, 4 and 6 are admissible, whose individual component trees map to 0-, 1- 2- and 3-periodic regular nets. The last cases form mutually entangled srs, dia and pcu nets, respectively, on the TPMS. In this paper, we have canvassed the patterns that emerge from the next simplest hyperbolic forests, namely regular rare examples. Here only 0 - and 2-periodic nets of degree 3 are possible. Despite the more limited net topologies that build the entangled patterns - namely $\theta$-graphs and hcb nets - an extraordinary variety of embeddings has been found. Some of those patterns are trivial (untangled), many others are catenated in ways recognized already in material framework structures, especially metal-organic frameworks. Further, it is clear that a variety of entanglement motifs are possible, from simpler Hopf and Borromean types, to ravels, and cases with complex features, such as hierarchical two- to threedimensional entanglements. In some (though not all) cases, those can be partially characterized by measures introduced by chemists to label tangled structures (Alexandrov et al., 2012). However, it is apparent from the more complex examples presented here 


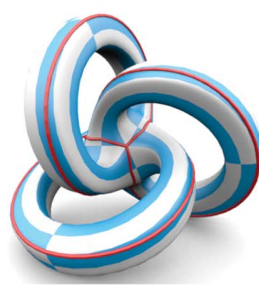

(a)

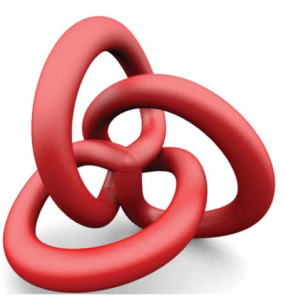

(b)

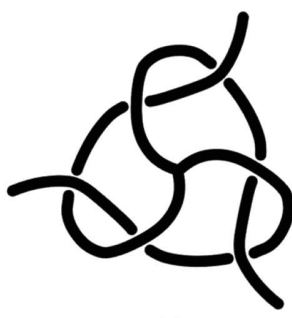

(c)
Figure 28

$(a) \star 246_{49 R T}\left(\cosh ^{-1}(399)\right)$ on the tritorus. (b) The embedded graph is a 3-ravel. (c) A planar representation, where one vertex has been split open.

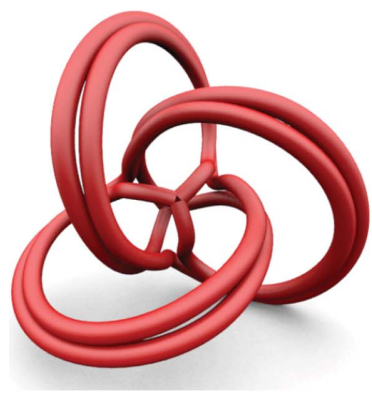

(a)

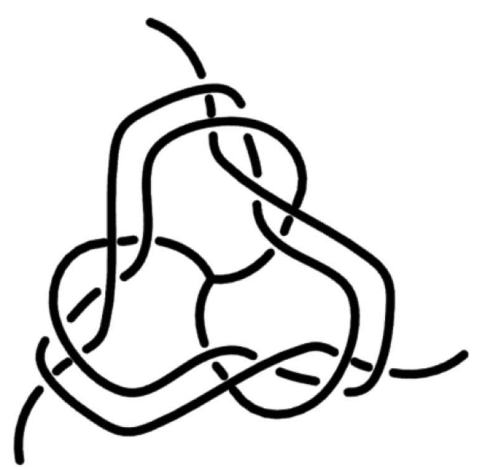

(b)
Figure 29

(a) The graph embedding resulting from the compactification of $\star 246_{49 R T}\left(\cosh ^{-1}(3233)\right)$ to the tritorus. (b) A simpler representation of this embedded graph, where a vertex has been split for simplicity.

that much work remains to properly characterize generic entangled nets, even when the components are very simple layer structures, such as the hcb net.

Lastly, we note that there remain a number of other TPMSs that can be used as supports for such reticulations. Certainly, the genus-3 TPMSs are the simplest, and will give the most symmetric regular structures. One other genus-3 surface with distinct compactified structure is known, namely the tetragonal CLP surface (Hyde et al., 2014). It turns out that this case does not yield novel examples of tangled patterns beyond those explored in the paper. We therefore assert that the most symmetric examples of tangled patterns related to regular hyperbolic forests are now fully known.

\section{References}

Alexandrov, E. V., Blatov, V. A. \& Proserpio, D. M. (2012). Acta Cryst. A68, 484-493.

Batten, S. R. \& Robson, R. (1998). Angew. Chem. Int. Ed. 37, 14601494.

Blatov, V. A., Carlucci, L., Ciani, G. \& Proserpio, D. M. (2004). CrystEngComm, 6, 377-395.

Blatov, V., Shevchenko, A. \& Proserpio, D. (2014). Cryst. Growth Des. 14, 3576-3586.

Bonneau, C. \& O'Keeffe, M. (2015). Acta Cryst. A71, 82-91.

Carlucci, L., Ciani, G. \& Proserpio, D. M. (2003a). CrystEngComm, 5, 269-279.

Carlucci, L., Ciani, G. \& Proserpio, D. M. (2003b). Coord. Chem. Rev. 246, 247-289.
Carlucci, L., Ciani, G., Proserpio, D. M., Mitina, T. G. \& Blatov, V. A. (2014). Chem. Rev. 114, 7557-7580.

Castle, T., Evans, M. E. \& Hyde, S. T. (2008). New J. Chem. 32, 1484 1492.

Castle, T., Evans, M. E. \& Hyde, S. T. (2011). Prog. Theor. Phys. Suppl. 191, 235-244.

Castle, T., Evans, M. E., Hyde, S. T., Ramsden, S. J. \& Robins, V. (2012). Interface Focus, 2, 555-566.

Chen, B., Eddaoudi, M., Hyde, S., O'Keeffe, M. \& Yaghi, O. (2001). Science, 291, 994-1021.

Chung, S. J., Hahn, Th. \& Klee, W. E. (1984). Acta Cryst. A40, 42-50.

Conway, J. (1992). Groups, Combinatorics and Geometry. London Mathematical Society Lecture Note Series 165. Cambridge University Press.

Conway, J. H. \& Huson, D. H. (2002). Struct. Chem. 137, 247-257.

Delgado-Friedrichs, O. (2003). Theor. Comput. Sci. 303, 431-445.

Delgado-Friedrichs, O. \& Huson, D. (1999). Discrete Comput. Geom. 21, 299-315.

Delgado-Friedrichs, O. \& O'Keeffe, M. (2003). Acta Cryst. A59, 351360.

Delgado-Friedrichs, O. \& O'Keeffe, M. (2005). J. Solid State Chem. 178, 2480-2485.

Delgado Friedrichs, O., O'Keeffe, M. \& Yaghi, O. M. (2003). Acta Cryst. A59, 22-27.

Emmer, M. \& Schattschneider, D. (2003). M. C. Escher's Legacy: a Centennial Celebration. Berlin, Heidelberg: Springer-Verlag.

Eon, J.-G. (2005). Acta Cryst. A61, 501-511.

Evans, M. E., Robins, V. \& Hyde, S. T. (2013a). Acta Cryst. A69, 241261.

Evans, M. E., Robins, V. \& Hyde, S. T. (2013b). Acta Cryst. A69, 262275.

Evans, M. E., Robins, V. \& Hyde, S. T. (2015). Proc. R. Soc. London Ser. A, 471, 2015025.

Fischer, W. \& Koch, E. (1976). Acta Cryst. A32, 225-232.

Han, Y., Zhang, D., Chng, L. L., Sun, J., Zhao, L., Zou, X. \& Ying, J. Y. (2009). Nat. Chem. 1, 123-127.

Huson, D. H. (1993). Geom. Dedicata, 47, 269-296.

Hyde, S. T., de Campo, L. \& Oguey, C. (2009). Soft Matter, 5, 27822794.

Hyde, S. T. \& Oguey, C. (2000). Euro. Phys. J. B, 16, 613-630.

Hyde, S. T. \& Ramsden, S. (2000). Europhys. Lett. 50, 135-141.

Hyde, S. T., Ramsden, S. J. \& Robins, V. (2014). Acta Cryst. A70, 319337.

Hyde, S., Robins, V. \& Ramsden, S., (2015). Epinet. http:// epinet.anu.edu.au.

Kirkensgaard, J. J. K., Evans, M. E., de Campo, L. \& Hyde, S. T. (2014). Proc. Natl Acad. Sci. USA, 111, 1271-1276.

Koch, E., Fischer, W. \& Sowa, H. (2006). Acta Cryst. A62, 152-167.

Li, F., Clegg, J. K., Lindoy, L. F., Macquart, R. B. \& Meehan, G. V. (2011). Nat. Commun. 2, 205.

Miller, J. S. (2001). Adv. Mater. 13, 525-527.

O'Keeffe, M. (1991). Acta Cryst. A47, 748-753.

O'Keeffe, M., Peskov, M. A., Ramsden, S. J. \& Yaghi, O. (2008). Acc. Chem. Res. 41, 1782-1789.

Ramsden, S. J., Robins, V. \& Hyde, S. T. (2009). Acta Cryst. A65, 81108.

Reineke, T. M., Eddaoudi, M., Moler, D., O'Keeffe, M. \& Yaghi, O. M. (2000). J. Am. Chem. Soc. 122, 4843-4844.

Robins, V. (2015). http://people.physics.anu.edu.au/ vbr110/ PDGdata/.

Robins, V., Ramsden, S. \& Hyde, S. (2004a). Physica A, 339, 173-180.

Robins, V., Ramsden, S. J. \& Hyde, S. T. (2004b). Euro. Phys. J. B, 39, 365-375.

Robins, V., Ramsden, S. \& Hyde, S. (2005). Euro. Phys. J. B, 48, 107111.

Schattschneider, D. (2004). M. C. Escher: Visions of Symmetry. London: Thames and Hudson Ltd.

Schoen, A. H. (1970). NASA Technical Note TN D-5541. 
Schröder-Turk, G., de Campo, L., Evans, M. E., Saba, M., Kapfer, S. C., Varslot, T., Grosse-Brauckmann, K., Ramsden, S. \& Hyde, S. (2013). Faraday Discuss. 161, 215-247.

Sowa, H. (2009). Acta Cryst. A65, 326-327.
Thurston, W. (1980). The Geometry and Topology of ThreeManifolds. Princeton University Press.

Wells, A. (1977). Three-Dimensional Nets and Polyhedra. New York: John Wiley and Sons. 